Biomath Forum

\title{
Modelling and Analysis of miRNA Regulation
}

\author{
Svetoslav G. Nikolov \\ Institute of Mechanics, Bulgarian Academy of Sciences, Bulgaria \\ Department of Systems Biology and Bioinformatics, University of Rostock \\ University of Transport, Sofia, Bulgaria \\ e-mail: S.Nikolov@imbm.bas.bg
}

Received: 16 May 2014, accepted: 23 July 2014, published: 15 September 2014

\begin{abstract}
MiRNA regulation is involved in many important biological processes such as cell proliferation, apoptosis and metabolism. Computational predictions of miRNA targets suggest that up $30 \%$ of human proteins coding genes may be regulated by miRNAs. This makes miRNAs one of the most abundant classes of regulatory genes in humans.

In the present paper we develop a time delay model of a feedback system regulated via miRNA. The model resulted in three DDEs with three discrete time delays. Since this system is a classical case study, covering several essential features of miRNA and genetic regulatory mechanisms, general conclusions about design principles and role of time delays in the stability of gene circuits can be suggested. The basic view that time delays are a key factor in the dynamic behaviour of the system was confirmed by the analytical calculations and numerical simulations.
\end{abstract}

Keywords-miRNA regulation; time delay; Andronov-Hopf bifurcation; numerical analysis

\section{INTRODUCTION}

Cells are the structural and functional units of all living organisms as protein synthesis is an essential function of a cell. Protein synthesis is achieved in a two-step process. Firstly, when a protein is needed in a cell, a messenger RNA (mRNA) is created as a copy of the information from the appropriate gene (process called transcription). Then, the mRNA molecule is used as a template for the creation of the protein (process called translation). The processes of transcription and translation in eukaryotic cells are very complicated, since there are more levels of control of gene expression. Transcription and translation are processes which require a certain amount of time to complete. Thus, these two processes of gene expression induce time delays in the biochemical systems.

Smolen and coauthors in [28] describe a time delay associated to the translocation of proteins and mRNAs between 50 and 100 min. Rateitschak and Wolkenhauer in [24] describe a time delay for gene transcription between 10 and $40 \mathrm{~min}$. Finally in [29] a time delay around $7 \mathrm{~min}$ is defined and estimated for nucleocytoplasmic shuttling.

Recently, an additional level of regulation in protein expression has been introduced following the identification of short non-protein-coding RNAs [22]. MicroRNAs (miRNAs) are small regulatory RNAs with length of 18-25 nucleotides (typically $\approx 22$ nt size in human), which function 
is to regulate the activity and stability of specific messenger RNA targets. A large body of data suggests that miRNAs are supposed to account for $1-5 \%$ of animal genes [3, 12] (bind through imperfect base paining to the 3' UTR of their target mRNAs and interfere with translational output [16]), making them one of the most abundant gene product regulators.

MiRNAs are embedded in complex regulatory networks that involved gene activation, posttranslational regulation and protein-protein interactions. Therefore, this makes miRNAs as one of the most abundant classes of regulatory genes in animals. A key recurring function of miRNA in networks is to reinforce the gene expression of differentiated cellular states. For example, regulatory networks involving miRNAs have been described in the asymmetric differentiation of left-right neurons in C.elegans, eye and sensory organprecursor development in Drosophila, and granulocytic differentiation in human [8]. Thus, miRNA provide an ideal way to regulate rapid and localized protein synthesis. A recent study [31] classified gene regulatory networks involving miRNAs in two types of circuits: in type A circuits the transcription of the miRNAs and their targets are positively coregulated, while in type $\mathrm{B}$ circuits the transcription of the miRNAs and their targets is oppositely regulated by common upstream factors or processessee Fig.1. Type A circuits may be more prevalent in networks operating in homeostasis, to maintain in protein steady state and in general to reduce the noise in transcriptional/translational fluctuations. Type B circuits instead can serve as surveillance mechanisms to suppress 'leaky' transcription of target gene, and in general as positive feedback loops where a transient signal can be converted in a long-lasting cellular response such as irreversible cellular differentiation [8].

Time lags in continuous systems can produce complex dynamics and instabilities. In the recent years ordinary differential equations (ODEs) with retarded argument(s) have been widely used in modelling and analyzing regulatory systems involve many genes, factors and complex interac- tions (Gene Regulatory Networks). In these models, the gene and mRNA concentrations are timedependent variables, interactions are represented by functional and differential between variables, and retarded arguments are usually the time of transcription and translation, or time delay feedback loop. For the concentration $x_{i}(t)$ of the factor $i$ at time $t$, the evolution in time is described by the system:

$$
\begin{aligned}
& \frac{d x_{i}(t)}{d t}=a x_{i}(t)+b x_{i}(t-\tau)= \\
& f_{i}\left(x_{j}(t), x_{j}(t-\tau), j \in N_{i}\right)(i=1,2, \ldots, n),
\end{aligned}
$$

where $f_{i}$ is a nonlinear real-valued function of the states $\left(x_{j}(t), x_{j}(t-\tau)\right)$ of the vertices $j \in N_{i}$, that interact with the factor $i$. An interesting and important problem appears due to the nonlinearity of $f_{i}$, since an analytical solution of the system is usually not possible.

In this sense, the recent molecular biological discoveries (like the miRNAs and their complex regulatory effects) clearly show the need to develop mathematical models that take into consideration the post-transcriptional regulation. Also, the models should take into account that the interactions can be complex, consisting of cyclic dependences, cooperative regulations (including Transcription Factors (TFs) that act simultaneously or that assemble in complex structures), DNA looping regulation, etc. These criteria seem difficult to satisfy with a standard modeling formalism, and answers may lie in hybrid approaches [11].

As an intuitive example of nonlinearity by characterizing the behavior of a system in terms of stimulus and response is the following: If we give the system a "kick" or "input signal" and observe a certain response to that kick, then we can ask what happens if we kick the system twice as hard. If the response is not twice as large (it might be larger or smaller), then we say the system's behavior is nonlinear [7]. What is the fuss over nonlinearity? The basic idea is that, for nonlinear systems, a small change in a parameter can lead to sudden and dramatic changes in both the qualitative and 


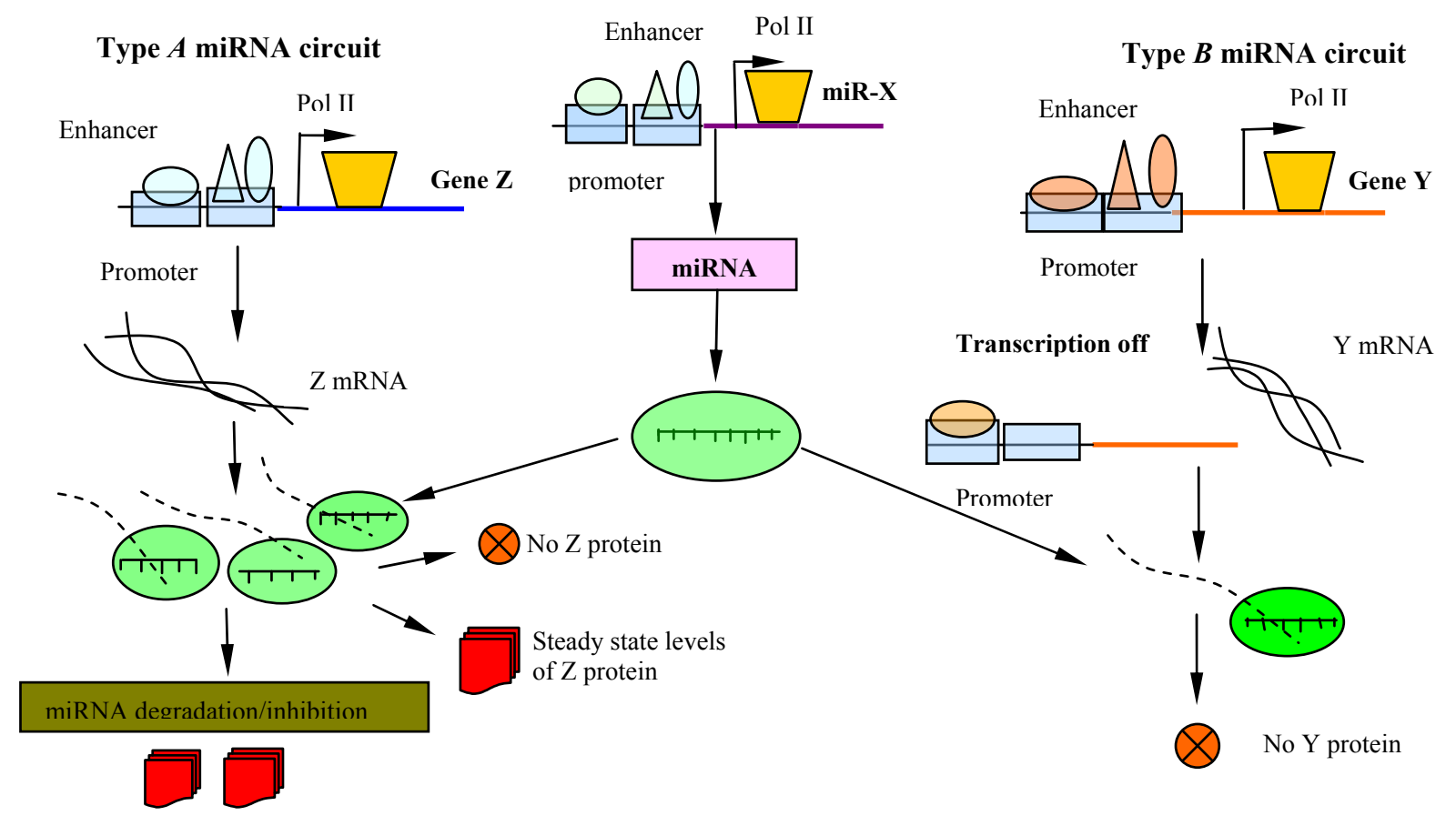

Fig. 1. Integrating miRNAs into regulatory networks. Type A circuits might be involved in maintaining protein steady-state levels and homeostasis. Type B circuits can serve as a surveillance mechanism to suppress leaky transcription of target genes.

quantitative behavior of the system. For one value, the behavior might be stable, for another value only slightly different from the first, the behavior might be periodic or completely aperiodic.

When time-delay, feedback-loops and nonlinearity appear combined in systems like miRNA networks, a suitable approach for investigating its dynamics is the systems bifurcation analysis. Bifurcation theory studies persistence and exchange of qualitative properties of dynamical systems under continuous perturbations. From the point of view of the dynamic systems theory, the Hopf bifurcation theorem [22] together with other elements of the bifurcation theory are basic analytical tools to investigate pathological conditions in biological systems.

A typical case is that of systems depending continuously on a single parameter. Under small variations of the parameter, the systems may lose stability, and re-stabilize near another equilibrium or a closed orbit, or a larger attractor. The type of transition can be continuous, gentle and smooth, with the new equilibrium being far from the "trivial" one [2, 27]. The first case corresponds to a local bifurcation, the second one is a global bifurcation. Local bifurcations can be of two types: i) the system leaves its equilibrium state and reaches a new equilibrium state; ii) the system goes from an equilibrium state to an invariant subset generally composed of several equilibriums and curves connecting them, closed orbits, or tori, etc. For example, two component mechanisms with autocatalysis easily generate oscillations and bistability. They also exhibit a rich structure of bifurcations to more complicated behavior, for example pitchfork bifurcation, saddle-node bifurcation or Takens-Bogdanov bifurcation [26]. The most popular and elementary situation is the AndronovHopf bifurcation, characterized by the onset of a closed orbit, starting near the trivial equilibrium (from focus type), which is the phase portrait of a periodic solution with a period close to some fixed 
number $[1,27]$. In this work, we will restrict our investigation to the Andronov-Hopf bifurcation.

The aim of this article is to elucidate how the dynamics of the gene expression (associated with the time delays) is regulated by the miRNA. Thus, the plan of the paper is as follows: In Section 2 we formulate the mathematical model. It results in three delayed differential equations (DDEs). A qualitative analysis of the model equations is performed in Section 3. In Section 4 we explore numerically the model. Finally, in Section 5, we discuss and unify results from previous sections.

\section{MODEL}

There are two possible ways in which miRNAs can play a role in gene expression [33]. One is that they can accelerate the degradation of mRNAs, while the other is that they repress translation by forming silencing complexes (see Fig. 2). In the version of the model discussed here we suppose that the system acts as a feedback loop where the protein, $y_{2}$, controls its own synthesis through the repression of mRNA, $y_{1}$. Here we construct one equation, describing the rate of change in the concentration of miRNA, $y_{3}$, taking into account only the first possible mechanism. We assume also (similarly to [33]) that the production of miRNA occurs at a constant rate, $l$, and we define $k_{6}$ as its degradation rate. According to the first mechanism (in which miRNAs are regarded as an enhancer of mRNA degradation) an extra degradation term, $k_{4} y_{1} y_{3}$, to the rate equation for mRNA and miRNA (where $k_{4}$ is the degradation rate) is added. Based on these hypotheses, the system can be represented by the following mathematical model in time delayed differential equations

$$
\begin{aligned}
\frac{d y_{1}}{d t} & =\frac{k_{1}}{k_{2}+k_{3} y_{2}^{n_{1}}\left(t-\tau_{1}\right)}-\gamma_{1} y_{1}-k_{4} y_{1} y_{3}, \\
\frac{d y_{2}}{d t} & =k_{5} y_{1}\left(t-\tau_{2}\right)-\gamma_{2} y_{2}, \\
\frac{d y_{3}}{d t} & =l-k_{6} y_{3}\left(t-\tau_{3}\right)-k_{4} y_{1} y_{3},
\end{aligned}
$$

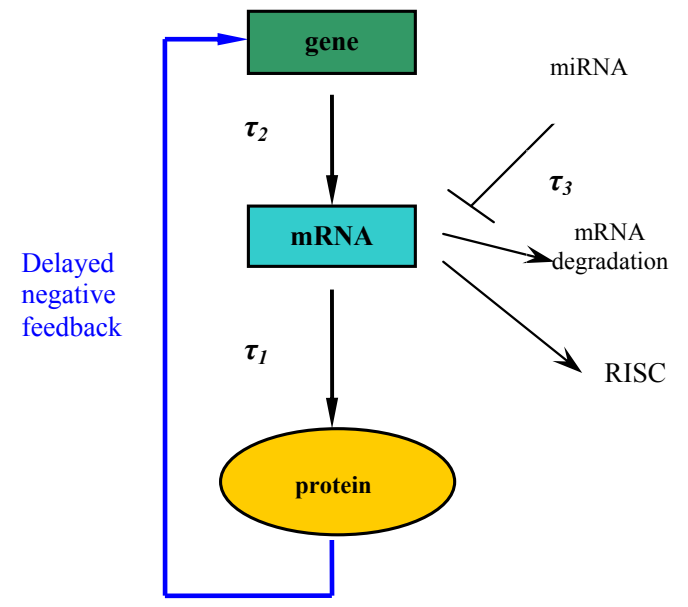

Fig. 2. Scheme of transcription $\left(\tau_{1}\right)$ and translation $\left(\tau_{2}\right)$ time delay negative feedback control of protein synthesis

where $\tau_{1}$ is the time delay for translation, $\tau_{2}$ is the time delay for transcription, $\tau_{3}$ is the average time delay for degradation of miRNA, $n_{1}$ is often referred as a Hill coefficient or a cooperativity coefficient, $k_{i}(i=1, \ldots, 6)$ and $\gamma_{1}, \gamma_{2}$ are the kinetic rate constants. In the present work, according to [20,34], we consider one representative value for Hill coefficient, $n_{1}=2$.

\section{Qualitative AnAlysis}

In this section, we consider the system (2) when the Hill coefficient, $n_{1}$, is equal to two and all constants of the model are real positive numbers. Furthermore, we investigate the bifurcation structure- particularly the Andronov-Hopf bifurcation- for system (2), using time delays $\tau_{1}, \tau_{2}$ or $\tau_{3}$ as bifurcation parameters. Here, we note that system (2) is a private case of the general system considered in [23], and for our analysis we use a specific theoretical approach obtained there.

The fixed points of the system, $\bar{E}=$ $\left(\bar{y}_{1}, \bar{y}_{2}, \bar{y}_{3}\right)$, represented by Eq. (2) can be analytically estimated and are defined by the following set of algebraic equations, including the rate constants of the model: 


$$
\begin{aligned}
& \bar{y}_{2}^{4}+\frac{k_{5}\left(k_{4} l+k_{6} \gamma_{1}\right)}{k_{4} \gamma_{1} \gamma_{2}} \bar{y}_{2}^{3}+\frac{k_{2}}{k_{3}} \bar{y}_{2}^{2} \\
& +\frac{k_{5}}{k_{3} k_{4} \gamma_{1} \gamma_{2}}\left[k_{2}\left(k_{4} l+k_{6} \gamma_{1}\right)-k_{1} k_{4}\right] \bar{y}_{2} \\
& -\frac{k_{1} k_{5}^{2} k_{6}}{k_{3} k_{4} \gamma_{1} \gamma_{2}^{2}}=0, \bar{y}_{1}=\frac{\gamma_{2}}{k_{5}} \bar{y}_{2}, \\
& \bar{y}_{3}=\frac{k_{5} l}{k_{5} k_{6}+\gamma_{2} k_{4} \bar{y}_{2}} .
\end{aligned}
$$

According to Descarte's rule [10, 14], the first equation in (3) has only one real positive root, which ensures that system has only one physiologically feasible fixed point.

Let us consider a small perturbation around the fixed point $\bar{E}$ of the system (2) defined as:

$$
y_{k}=\bar{y}_{k}+x_{k} \quad(k=1,2,3)
$$

In the case when $n_{1}=2$, the function $\frac{k_{1}}{k_{2}+k_{3} y_{2}^{2}\left(t-\tau_{1}\right)}$ can be written as a MacLaurin series:

$$
\begin{aligned}
& \frac{k_{1}}{k_{2}+k_{3} y_{2}^{2}\left(t-\tau_{1}\right)}=\frac{k_{1}}{\delta+k_{3} \eta}=\frac{k_{1}}{\delta\left(\frac{k_{3}}{\delta} \eta+1\right)} \\
& =\frac{k_{1}}{\delta}\left(1-\frac{k_{3}}{\delta} \eta+\left(\frac{k_{3}}{\delta}\right)^{2} \eta^{2}-\left(\frac{k_{3}}{\delta}\right)^{3} \eta^{3}+\ldots\right)
\end{aligned}
$$

where $\delta=k_{2}+k_{3} \bar{y}_{2}^{2}$ and $\eta=2 \bar{y}_{2} x_{2}\left(t-\tau_{1}\right)+$ $x_{2}^{2}\left(t-\tau_{1}\right)$. If we take only linear term from (5) and after substitution of (4) into differential equation (2) we have

$$
\begin{aligned}
\frac{d x_{1}}{d t}= & -c_{1} x_{1}-c_{2} \ell^{-\chi \tau_{1}} x_{2}-c_{3} x_{3}-c_{4} \ell^{-2 \chi \tau_{1}} x_{2}^{2} \\
& -k_{4} x_{1} x_{3} \\
\frac{d x_{2}}{d t}= & k_{5} \ell^{-\chi \tau_{2}} x_{1}-\gamma_{2} x_{2} \\
\frac{d x_{3}}{d t}= & -c_{5} x_{1}-\left(k_{6} \ell^{-\chi \tau_{3}}+c_{3}\right) x_{3}-k_{4} x_{1} x_{3}
\end{aligned}
$$

where

$$
\begin{aligned}
& c_{1}=\gamma_{1}+k_{4} \bar{y}_{3}, c_{2}=\frac{2 k_{1} k_{3} \bar{y}_{2}}{\delta^{2}}, c_{3}=k_{4} \bar{y}_{1}, \\
& c_{4}=\frac{k_{1} k_{3}}{\delta^{2}}, c_{5}=k_{4} \bar{y}_{3} .
\end{aligned}
$$

The characteristic equation of (6) has the form

$$
\begin{aligned}
& \operatorname{det}\left(\begin{array}{ccc}
-c_{1}-\chi & -c_{2} \ell^{-\chi \tau_{1}} & -c_{3} \\
k_{5} \ell^{-\chi \tau_{2}} & -\gamma_{2}-\chi & 0 \\
-c_{5} & 0 & -\left(c_{3}+k_{6} \ell^{-\chi \tau_{3}}\right)-\chi
\end{array}\right) \\
& =0
\end{aligned}
$$

that is

$$
\begin{aligned}
& \chi^{3}+K_{1} \chi^{2}+K_{2} \chi+K_{3}=\ell^{-\chi \tau_{3}}\left(T_{1} \chi^{2}+T_{2} \chi\right. \\
& \left.+T_{3}\right)+\ell^{-\chi\left(\tau_{1}+\tau_{2}\right)}\left(T_{4} \chi+T_{5}\right)+T_{6} \ell^{-\chi\left(\tau_{1}+\tau_{2}+\tau_{3}\right)}
\end{aligned}
$$

where

$$
\begin{aligned}
& K_{1}=\gamma_{2}+c_{1}+c_{3} \\
& K_{2}=\gamma_{2} c_{1}+c_{3}\left(\gamma_{2}+c_{1}-c_{5}\right) \\
& K_{3}=\gamma_{2} c_{3}\left(c_{1}-c_{5}\right), T_{1}=-k_{6} \\
& T_{2}=-k_{6}\left(\gamma_{2}+c_{1}\right), T_{3}=-\gamma_{2} k_{6} c_{1} \\
& T_{4}=-k_{5} c_{2}, T_{5}=-k_{5} c_{2} c_{3}, T_{6}=-k_{5} k_{6} c_{2} .
\end{aligned}
$$

Remark 1. We note that $\chi=0$ is a root of (9) if and only if $K_{3}=T_{3}=T_{5}=T_{6}=0$.

In the absence of delays ( $\tau_{1}=\tau_{2}=\tau_{3}=0$ ), $\bar{E}$ is locally asymptotically stable if

$$
\begin{aligned}
& p=\gamma_{2}+k_{6}+c_{1}+c_{3}>0 \\
& q=\gamma_{2}\left(k_{6}+c_{1}+c_{3}\right)+k_{5} c_{2}+c_{3}\left(c_{1}-c_{5}\right)>0, \\
& r=\gamma_{2} c_{3}\left(c_{1}-c_{5}\right)+\gamma_{2} c_{1} k_{6}+k_{5} c_{2}\left(k_{6}+c_{3}\right)>0 \\
& R=p q-r>0 .
\end{aligned}
$$


Here we note that (9) is a third-degree exponential polynomial in $\chi$ with three discrete time delays. Because of the presence of three different discrete delays into (9), the analysis of the sign of the real parts of the eigenvalues is very complicated, and a direct approach cannot be considered. Thus, in our analysis we will use a method consisting of determining the stability of the steady state when firstly two delays are equal to zero, and secondly when one delay is equal to zero.

The case $\tau_{1}=\tau_{3}=0, \tau_{2}>0$.

Generally, in eukariotes translation takes longer than transcription and one of the reasons is intron splicing. It has been suggested that the length of the introns is fundamental to cell timing [30]. Hence, we assume that the finite time delay $\tau_{2}$ of transcription is longer than the time delay $\tau_{1}$ of translation and finite time delay $\tau_{3}$ of degradation of miRNA.

Setting $\tau_{1}=\tau_{3}=0$ into (9), the characteristic equation becomes

$\chi^{3}+K_{11} \chi^{2}+K_{21} \chi+K_{31}=\ell^{-\chi \tau_{2}}\left(T_{4} \chi+T_{51}\right)$,

where

$$
\begin{aligned}
& K_{11}=K_{1}-T_{1}, K_{21}=K_{2}-T_{2}, \\
& K_{31}=K_{3}-T_{3}, T_{51}=T_{5}+T_{6} .
\end{aligned}
$$

It is well-known that that the stability of the equilibrium state $\bar{E}$ depends on the sign of the real parts of the roots of (12). We recall that a steady state is locally asymptotically stable if and only if all roots of (12) have negative real parts, and its stability can only be lost if these roots cross the vertical axis, that is if purely imaginary roots appear. Generally speaking, the transcendental equation (12) (for nonzero delay) cannot be solved analytically and has an indefinite number of roots. In essence, we have two main tools besides direct numerical integration; firstly, the linear stability analysis in the case of a small time delay, and secondly, the Hopf bifurcation theorem. Because from biological point of view it is known that time delay of transcription, $\tau_{2}$, in many cases is bigger than one [15, 20, 21, 35] here we use Hopf bifurcation theorem. Thus, we let $\chi=m+i n(m, n \in R)$, and rewrite (12) in terms of its real and imaginary parts as

$$
\mid \begin{aligned}
& m^{3}-3 m n^{2}+K_{11}\left(m^{2}-n^{2}\right)+K_{21} m+K_{31} \\
& =\ell^{-m \tau_{2}}\left[\left(T_{4} m+T_{51}\right) \cos n \tau_{2}+T_{4} n \sin n \tau_{2}\right] \\
& -n^{3}+3 m^{2} n+2 K_{11} m n+K_{21} n \\
& =\ell^{-m \tau_{2}}\left[T_{4} n \cos n \tau_{2}-\left(T_{4} m+T_{51}\right) \sin n \tau_{2}\right] .
\end{aligned}
$$

To find the first bifurcation point we look for purely imaginary roots $\chi= \pm i n, n \in R$ of (12), i.e. we set $m=0$. Then the above two equations are reduced to

$$
\mid \begin{aligned}
& -K_{11} n^{2}+K_{31}=T_{51} \cos n \tau_{2}+T_{4} n \sin n \tau_{2}, \\
& -n^{3}+K_{21} n=T_{4} n \cos n \tau_{2}-T_{51} \sin n \tau_{2},
\end{aligned}
$$

or another one

$\begin{aligned} \cos n \tau_{2} & =\frac{T_{51}\left(K_{31}-K_{11} n^{2}\right)+T_{4} n^{2}\left(K_{21}-n^{2}\right)}{T_{51}^{2}+T_{4}^{2} n^{2}} \\ \sin n \tau_{2} & =\frac{n\left[T_{4}\left(K_{31}-K_{11} n^{2}\right)-T_{51}\left(K_{21}-n^{2}\right)\right]}{T_{51}^{2}+T_{4}^{2} n^{2}} .\end{aligned}$

It is clear that if the first bifurcation point is $\left(n_{b}^{0}, \tau_{b}^{0}\right)$, then the other bifurcation points $\left(n_{b}, \tau_{b}\right)$ satisfy $n_{b} \tau_{b}=n_{b}^{0} \tau_{b}^{0}+2 \nu \pi, \nu=1,2, \ldots, \infty$.

One can notice that if $n$ is a solution of (15) (or (16)), then so is $-n$. Hence, in the following we only investigate for positive solutions $n$ of (15), or (16) respectively. By squaring the two equations into system (15) and then adding them, it follows that: 


$$
\begin{aligned}
& n^{6}+\left(K_{11}^{2}-2 K_{21}\right) n^{4}+\left(K_{21}^{2}-2 K_{11} K_{31}\right. \\
& \left.-T_{4}^{2}\right) n^{2}+K_{31}^{2}-T_{51}^{2}=0
\end{aligned}
$$

As $\bar{E}$ is locally asymptotically stable at $\tau_{2}=0$, satisfies the Routh-Hurwitz conditions for stability for a cubic polynomial [9, 19]. Equation (17) is a cubic in $n^{2}$ and the left-hand side is positive for very large values of $n^{2}$ and negative for $n=0$ if and only if $T_{51}^{2}>K_{31}^{2}$, i.e. when Eq. (17) has at least one positive real root. Moreover, to apply the Hopf bifurcation theorem, according to [9], the following theorem in this situation applies:

Theorem 1. Suppose that $n_{b}$ is the last positive simple root of (17). Then, in $\left(\tau_{b}\right)=i n_{b}$ is a simple root of (12) and $m\left(\tau_{2}\right)+i n\left(\tau_{2}\right)$ is differentiable with respect to $\tau_{2}$ in a neighbourhood of $\tau_{2}=\tau_{b}$.

To establish an Andronov-Hopf bifurcation at $\tau_{2}=\tau_{b}$, we need to show that a pair of complex eigenvalues crosses the imaginary axis with nonzero speed, i.e. the following transversality condition $\left.\frac{d(\operatorname{Re} \chi)}{\mathrm{dt}}\right|_{\tau=\tau_{b}} \neq 0$ is satisfied.

From (15) we know that $\tau_{b_{k}}$ corresponding to $n_{b}$ is

$$
\begin{aligned}
& \tau_{b_{k}}=\frac{1}{n_{b}} \arccos \left[\left(-T_{4} n_{b}^{4}+\left(T_{4} K_{21}-T_{51} K_{11}\right) n_{b}^{2}\right.\right. \\
& \left.\left.+T_{51} K_{31}\right) /\left(T_{51}^{2}+T_{4}^{2} n_{b}^{2}\right)\right]+\frac{2 k \pi}{n_{b}} \\
& k=0,1,2, \ldots
\end{aligned}
$$

Because for $\tau_{2}=0$, equilibrium $\bar{E}$ is stable, by Butler's lemma [5], equilibrium remains stable for $\tau_{2}<\tau_{b_{k}}$, where $\tau_{b}=\tau_{b_{k}}$ as $k=0$. We have now to show that $\left.\frac{d(\operatorname{Re} \chi)}{\mathrm{dt}}\right|_{\tau=\tau_{b}} \neq 0$.

Hence, if denote

$$
\begin{aligned}
& H\left(\chi, \tau_{2}\right)=\chi^{3}+K_{11} \chi^{2}+K_{21} \chi+K_{31} \\
& -\ell^{-\chi \tau_{2}}\left(T_{4} \chi+T_{51}\right)
\end{aligned}
$$

then

$$
\begin{aligned}
& \frac{d \chi}{d \tau_{2}}=-\frac{\partial H}{\partial \tau_{2}} / \frac{\partial H}{\partial \chi}=\left(-\chi \ell^{-\chi \tau_{2}}\left(T_{4} \chi+T_{51}\right)\right) \\
& /\left(3 \chi^{2}+2 K_{11} \chi+K_{21}+\tau_{2} \ell^{-\chi \tau_{2}}\left(T_{4} \chi+T_{51}\right)\right. \\
& \left.-\ell^{-\chi \tau_{2}} T_{4}\right) .
\end{aligned}
$$

Evaluating the real part of this equation at $\tau_{2}=$ $\tau_{b}$ and setting $\chi=i n_{b}$ yield

$$
\begin{aligned}
& \left.\frac{d m}{d \tau_{2}}\right|_{\tau_{2}=\tau_{b}}=\left.\frac{d(\operatorname{Re} \chi)}{d t}\right|_{\tau_{2}=\tau_{b}} \\
& =\left(n _ { b } ^ { 2 } \left[3 n_{b}^{4}+2\left(K_{11}^{2}-2 K_{21}\right) n_{b}^{2}+K_{21}^{2}\right.\right. \\
& \left.\left.-2 K_{11} K_{31}-T_{4}^{2}\right]\right) /\left(L^{2}+I^{2}\right)
\end{aligned}
$$

where

$$
\begin{aligned}
L= & -3 n_{b}^{2}+K_{21}+\tau_{2}\left(-K_{11} n_{b}^{2}+K_{31}\right) \\
& -T_{4} \cos n_{b} \tau_{2} \\
I= & 2 K_{11} n_{b}+\tau_{2}\left(-n_{b}^{3}+K_{21} n_{b}\right)+T_{4} \sin n_{b} \tau_{2} .
\end{aligned}
$$

Let $\theta=n_{b}^{2}$, then, (17) reduces to

$$
\begin{aligned}
& g(\theta)=\theta^{3}+\left(K_{11}^{2}-2 K_{21}\right) \theta^{2} \\
& \quad+\left(K_{21}^{2}-2 K_{11} K_{31}-T_{4}^{2}\right) \theta+K_{31}^{2}-T_{51}^{2}
\end{aligned}
$$

Then, for $g^{\prime}(\theta)$ we have

$$
\begin{aligned}
& \left.g^{\prime}(\theta)\right|_{\tau_{2}=\tau_{b}}=\left.\frac{d g}{d \theta}\right|_{\tau_{2}=\tau_{b}}=3 \theta^{2}+2\left(K_{11}^{2}-2 K_{21}\right) \theta \\
& +K_{21}^{2}-2 K_{11} K_{31}-T_{4}^{2} .
\end{aligned}
$$

If $n_{b}$ is the least positive simple root of (17), then 


$$
\left.\frac{d g}{d \tau_{2}}\right|_{\theta=n_{b}^{2}}>0
$$

Hence,

$$
\left.\frac{d m}{d \tau_{2}}\right|_{\tau_{2}=\tau_{b}}=\left.\frac{d(\operatorname{Re} \chi)}{d \tau_{2}}\right|_{\tau_{2}=\tau_{b}}=\frac{n_{b}^{2} g^{\prime}\left(n_{b}^{2}\right)}{L^{2}+I^{2}}>0
$$

According to the Hopf bifurcation theorem [17], we define the following Theorem 2:

Theorem 2. If $n_{b}$ is the least positive root of (17), then an Andronov-Hopf bifurcation occurs as $\tau_{2}$ passes through $\tau_{b}$.

Corollary 2.1. When $\tau_{2}<\tau_{b}$, then the steady state $\bar{E}$ of system (2) is locally asymptotically stable.

The case $\tau_{1}=0 ; \tau_{2}, \tau_{3}>0$.

We return to the study of (9) which with $\tau_{2}, \tau_{3}>0$ has the form

$$
\begin{aligned}
& \chi^{3}+K_{1} \chi^{2}+K_{2} \chi+K_{3} \\
& =\ell^{-\chi \tau_{3}}\left(T_{1} \chi^{2}+T_{2} \chi+T_{3}\right)+\ell^{-\chi \tau_{2}}\left(T_{4} \chi+T_{5}\right) \\
& +T_{6} \ell^{-\chi \tau_{23}}
\end{aligned}
$$

where $\tau=\left[\tau_{2}, \tau_{3}, \tau_{23}=\tau_{2}+\tau_{3}\right]^{T}$ denotes a point in the time delay space, i.e. $\tau \in \Omega \subset R_{+}^{3} . \Omega$ is the time delay space and $R_{+}^{3}$ denotes the set of nonnegative real numbers. In order to assess the stability of $\bar{E}$ with respect to any delay $\tau$, one should know where all $\chi$ roots of (27) lie on the complex plane. Eq. (27) has infinitely many roots on the complex plane due to the transcendental term $\ell^{-\chi \tau}$. This makes the analytical stability assessment intractable.

Previously, we obtain that in the absence of delays, $\bar{E}$ is locally asymptotically stable if the conditions (11) are valid. By Remark 1, this implies that $\chi=0$ is not root of (27). Further, we introduce the following simple result (which is proved in [25]) using Rouche's theorem

Lemma 1. Consider the exponential polynomial $P\left(\chi, \ell^{-\chi \tau_{1}}, \ldots, \ell^{-\chi \tau_{m}}\right)=\chi^{n}+p_{1}^{(0)} \chi^{n-1}+\ldots$

$$
\begin{aligned}
& +p_{n-1}^{(0)} \chi+p_{n}^{(0)}+\left[p_{1}^{(1)} \chi^{n-1}+\ldots+p_{n-1}^{(1)} \chi\right. \\
& \left.+p_{n}^{(1)}\right] \ell^{-\chi \tau_{1}}+\ldots+\left[p_{1}^{(m)} \chi^{n-1}+\ldots+p_{n-1}^{(m)} \chi\right. \\
& \left.+p_{n}^{(m)}\right] \ell^{-\chi \tau_{m}}
\end{aligned}
$$

where $\tau_{i} \geq 0 \quad(i=1,2, \ldots, m)$ and $p_{j}^{(i)} \quad(i=0,1, \ldots, m ; j=1,2, \ldots, n) \quad$ are constants. As $\left(\tau_{1}, \tau_{2}, \ldots, \tau_{m}\right)$ vary, the sum of the order of the zeros of $P\left(\chi, \ell^{-\chi \tau_{1}}, \ldots, \ell^{-\chi \tau_{m}}\right)$ on the open right half plane can change only if a zero appears on or crosses the imaginary axis.

Obviously, in $(n>0)$ is a root of (27) if and only if $n$ satisfies

$$
\begin{aligned}
& -n^{3} i-K_{1} n^{2}+K_{2} n i+K_{3} \\
& =\left(\cos n \tau_{3}-i \sin n \tau_{3}\right)\left(-T_{1} n^{2}+T_{2} n i+T_{3}\right) \\
& +\left(\cos n \tau_{2}-i \sin n \tau_{2}\right)\left(T_{4} n i+T_{5}\right) \\
& +T_{6}\left(\cos n \tau_{23}-i \sin n \tau_{23}\right) .
\end{aligned}
$$

Separating the real and imaginary parts into (28), we obtain

$$
\mid \begin{aligned}
& -K_{1} n^{2}+K_{3}=T_{4} n \sin n \tau_{2}+T_{5} \cos n \tau_{2} \\
& \quad+\left(-T_{1} n^{2}+T_{3}\right) \cos n \tau_{3}+T_{2} n \sin n \tau_{3} \\
& \quad+T_{6} \cos n \tau_{23} \\
& \\
& -n^{3}+K_{2} n=T_{4} n \cos n \tau_{2}-T_{5} \sin n \tau_{2} \\
& -\left(-T_{1} n^{2}+T_{3}\right) \sin n \tau_{3}+T_{2} n \cos n \tau_{3} \\
& -T_{6} \sin n \tau_{23} .
\end{aligned}
$$

We square and add the equations (29), and after simplifying, we get that $\tau$ and $n$ must be 
among the real solutions of

$$
\begin{aligned}
& n^{6}+\left(K_{1}^{2}-T_{1}^{2}-2 K_{2}\right) n^{4}+\left(K_{2}^{2}-T_{2}^{2}-T_{4}^{2}\right. \\
& \left.-2 K_{1} K_{3}+2 T_{1} T_{3}\right) n^{2}+T_{6}^{2}+K_{3}^{2}-T_{3}^{2}-T_{5}^{2} \\
& =2\left\{\left[T_{2} T_{4} n^{2}+T_{5}\left(T_{1} n^{2}+T_{3}\right)\right] \cos n\left(\tau_{2}-\tau_{3}\right)\right. \\
& +n\left[T_{4}\left(-T_{1} n^{2}+T_{3}\right)-T_{2} T_{5}\right] \sin n\left(\tau_{2}-\tau_{3}\right) \\
& -T_{6}\left[\left(K_{1} n^{2}-K_{3}\right) \cos n \tau_{23}\right. \\
& \left.\left.+\left(-n^{3}+K_{2} n\right) \sin n \tau_{23}\right]\right\}
\end{aligned}
$$

We note that the right-hand side of (30) is always less than

$2\left[\left|T_{2} T_{4}\right| n^{2}+\left|T_{5}\right|\left|-T_{1} n^{2}+T_{3}\right|+\right.$ $\left(\left|T_{4}\right|\left|-T_{4} n^{2}+T_{3}\right|-\left|T_{2} T_{5}\right|\right) n-$ $\left.\left|T_{6}\right|\left(\left|K_{1} n^{2}-K_{3}\right|+\left|-n^{3}+K_{2} n\right|\right)\right]$.

Hence if the inequality

$$
\begin{aligned}
& \omega^{6}+\left(K_{1}^{2}-T_{1}^{2}-2 K_{2}\right) \omega^{4} \\
& \quad+\left(K_{2}^{2}-T_{2}^{2}-T_{4}^{2}-2 K_{1} K_{3}+2 T_{1} T_{3}\right) \omega^{2} \\
& \quad+T_{6}^{2}+K_{3}^{2}-T_{3}^{2}-T_{5}^{2}>2\left[\left|T_{2} T_{4}\right| \omega^{2}\right. \\
& \quad+\left|T_{5}\right|\left|-T_{1} \omega^{2}+T_{3}\right|+\left|T_{4}\right|\left|-T_{4} \omega^{2}+T_{3}\right| \omega \\
& \left.\quad-\left|T_{6}\right|\left(\left|K_{1} \omega^{2}-K_{3}\right|-\left|-\omega^{3}+K_{2} \omega\right|\right)\right]
\end{aligned}
$$

has no real solution on $0<\omega<n_{+}$, then (30) cannot be satisfied. Note that $n_{+}$is the positive solution of first equation in (29), which we write as

$$
\begin{aligned}
& K_{1} n^{2}=\psi(n)=\left[K+\left(T_{1} \cos n \tau_{3}\right) n^{2}\right. \\
& \quad-\left(T_{4} \sin n \tau_{2}+T_{2} \sin n \tau_{3}\right) n-T_{5} \cos n \tau_{2} \\
& \left.-T_{3} \cos n \tau_{3}-T_{6} \cos n \tau_{23}\right] \leq K_{3}+\left|T_{1}\right| n^{2} \\
& -\left(\left|T_{2}\right|+\left|T_{4}\right|\right) n-\left|T_{3}\right|-\left|T_{5}\right|-\left|T_{6}\right|,
\end{aligned}
$$

i.e.

$$
\begin{aligned}
& \left(K_{1}-\left|T_{1}\right|\right) n^{2}+\left(\left|T_{2}\right|+\left|T_{4}\right|\right) \\
& -\left(K_{3}-\left|T_{3}\right|-\left|T_{5}\right|-\left|T_{6}\right|\right)=0 .
\end{aligned}
$$

Thus, for $n_{+}$we have

$$
n_{+}=\frac{1}{2 a}\left(-b+\sqrt{b^{2}+4 \mathrm{ac}}\right)
$$

where $a=K_{1}-\left|T_{1}\right| \neq 0, b=\left|T_{2}\right|+\left|T_{4}\right|, c=$ $K_{3}-\left|T_{3}\right|-\left|T_{5}\right|-\left|T_{6}\right|$. It is clear that $n \leq n_{+}$.

Rearranging terms, we write (31) as

$$
\begin{aligned}
& \left(\omega\left|-\omega^{2}+K_{2}\right|-\left|T_{6}\right|\right)^{2}+\left(\left|K_{1} \omega^{2}-K_{3}\right|+\left|T_{6}\right|\right)^{2} \\
& \quad+\left(-T_{4} \omega^{2}+T_{3}\right)^{2}+\left(\left|T_{2}\right| \omega+\left|T_{5}\right|\right)+T_{4}^{2} \omega^{2}> \\
& \quad\left(\left|-T_{1} \omega^{2}+T_{3}\right|+\left|T_{5}\right|\right)^{2}+\left(\left|-T_{4} \omega^{2}+T_{3}\right|\right. \\
& \left.\quad+T_{4} \omega\right)^{2}+\left(T_{2}+T_{4}\right)^{2} \omega^{2}+T_{2}^{2} \omega^{2}+T_{5}^{2} .
\end{aligned}
$$

Hence, the following theorem can be formulated

Theorem 3. Let $K_{3}-\left|T_{3}\right|-\left|T_{5}\right|-\left|T_{6}\right| \neq 0$ and (34) hold. Then there is no change in stability of $\bar{E}$.

Remark 2. In the special case that $\tau_{2}=\tau_{3}$, the characteristic equation (27) becomes

$$
\begin{aligned}
& \chi^{3}+K_{1} \chi^{2}+K_{2} \chi+K_{3}= \\
& =\ell^{-\chi \tau_{2}}\left(T_{1} \chi^{2}+T_{24} \chi+T_{35}\right)+T_{6} \ell^{-2 \chi \tau_{2}}
\end{aligned}
$$

where $T_{35}=T_{3}+T_{5}$ and $T_{24}=T_{2}+T_{4}$. Therefore, this case is a private one of Theorem 3 .

Corollary 3.1. If conditions of Theorem 3 are not valid and $\tau_{2}^{\text {bif }}$ defined as in Theorem 2, then according to Lemma 1 for any $\tau_{2} \in\left[\begin{array}{ll}0, & \tau_{b}\end{array}\right)$, there exists a $\tau_{23}^{\text {bif }}\left(\tau_{2}\right)>0\left(\tau_{1}^{\text {bif }}\left(\tau_{2}\right)>0\right.$ respectively) such that the steady state $\bar{E}$ of system (2) is unstable when $\tau_{3} \in\left[0, \tau_{3}^{\text {bif }}\left(\tau_{2}\right)\right)$ ( $\tau_{1} \in\left[0, \tau_{1}^{\text {bif }}\left(\tau_{2}\right)\right)$ respectively), and an AndronovHopf bifurcation takes place.

The general case $\tau_{1}, \tau_{2}, \tau_{3}>0$.

Similar to previous Section, we set that $\chi=$ in $(n>0)$ is a root of (9) if and only if $n$ satisfies 


$$
\begin{aligned}
& n^{3} i-K_{1} n^{2}+K_{2} i n+K_{3} \\
& \quad=\left(\cos n \tau_{3}-i \sin n \tau_{3}\right)\left(-T_{1} n^{2}+T_{2} i n+T_{3}\right) \\
& \quad+\left(\cos n \tau_{4}-i \sin n \tau_{4}\right)\left(T_{4} i n+T_{5}\right) \\
& \quad+\left(\cos n \tau_{5}-i \sin n \tau_{5}\right) T_{6},
\end{aligned}
$$

where in this case $\tau=$ $\left[\tau_{3}, \tau_{4}=\tau_{1}+\tau_{2}, \tau_{5}=\tau_{1}+\tau_{2}+\tau_{3}\right]^{T}$ denotes a point in the time delay space, i.e. $\tau \in \Omega \subset R_{+}^{3}$. Here, $\Omega$ is the time delay space and $R_{+}^{3}$ denotes the set of nonnegative real numbers.

Separating the real and imaginary parts into (36), we have

$$
\mid \begin{aligned}
& -K_{1} n^{2}+K_{3}-A_{1}=B_{1}+T_{6} \cos n \tau_{5} \\
& -n^{3}+K_{2} n+A_{2}=B_{2}-T_{6} \sin n \tau_{5}
\end{aligned}
$$

where

$$
\begin{aligned}
& A_{1}=\left(-T_{1} n^{2}+T_{3}\right) \cos n \tau_{3}+T_{2} n \sin n \tau_{3}, \\
& A_{2}=\left(-T_{1} n^{2}+T_{3}\right) \sin n \tau_{3}-T_{2} n \cos n \tau_{3}, \\
& B_{1}=T_{4} n \sin n \tau_{4}+T_{5} \cos n \tau_{4}, \\
& B_{2}=T_{4} n \cos n \tau_{4}-T_{5} \sin n \tau_{4} .
\end{aligned}
$$

Adding up the squares of both equations into (37), we have

$$
\begin{aligned}
& n^{6}+\left(K_{1}^{2}+T_{1}^{2}-2 K_{2}\right) n^{4}+T_{3}^{2}-T_{5}^{2}-T_{6}^{2} \\
& \quad+\left(K_{2}^{2}+T_{2}^{2}-T_{4}^{2}-2 K_{1} K_{3}-2 T_{1} T_{3}\right) n^{2} \\
& \quad=2\left\{T_{6}\left(-T_{4} n \sin n \tau_{3}+T_{5} \cos n \tau_{3}\right)\right. \\
& \quad-\left(-n^{3}+K_{2} n\right)\left[\left(-T_{1} n^{2}+T_{3}\right) \sin n \tau_{3}\right. \\
& \left.\quad-T_{2} n \cos n \tau_{3}\right]-\left(K_{1} n^{2}-K_{3}\right)\left[\left(-T_{1} n^{2}+T_{3}\right)\right. \\
& \left.\left.\cos n \tau_{3}+T_{2} n \sin n \tau_{3}\right]\right\}
\end{aligned}
$$

where $\tau_{5}-\tau_{4}=\tau_{3}$. Clearly, the right-hand side of (38) is always less than

$$
\begin{aligned}
& 2\left[T_{6}\left|-T_{4} n+T_{5}\right|-\left(-n^{3}-K_{1} n^{2}+K_{2} n\right.\right. \\
& \left.\left.\quad+K_{3}\right)\left|-T_{1} n^{2}+T_{2} n+T_{3}\right|\right] .
\end{aligned}
$$

Hence if the inequality

$$
\begin{aligned}
& \lambda^{6}+\left(K_{1}^{2}+T_{1}^{2}-2 K_{2}\right) \lambda^{4} \\
& \quad+\left(K_{2}^{2}+T_{2}^{2}-T_{4}^{2}-2 K_{1} K_{3}-2 T_{1} T_{3}\right) \lambda^{2} \\
& \quad+T_{3}^{2}-T_{5}^{2}-T_{6}^{2}>2\left[T_{6}\left|-T_{4} \lambda+T_{5}\right|\right. \\
& \quad-\left(-\lambda^{3}-K_{1} \lambda^{2}+K_{2} \lambda+K_{3}\right) \mid-T_{1} \lambda^{2} \\
& \left.\quad+T_{2} \lambda+T_{3} \mid\right],
\end{aligned}
$$

has no real solution on $0<\lambda<n_{+}$, then (39) cannot be satisfied. Similar to previous Section, we note that $n_{+}$is the positive solution of first equation into (37), which is written as

$$
\begin{aligned}
& K_{1} n^{2}=\Psi(n)=K_{3}-\left[\left(-T_{1} n^{2}+T_{3}\right) \cos n \tau_{3}\right. \\
& \quad+T_{2} n \sin n \tau_{3}+T_{4} n \sin \tau_{4}+T_{5} \cos n \tau_{4} \\
& \left.+T_{6} \cos n \tau_{5}\right] \leq K_{3}+\left|T_{1}\right| n^{2}-\left(\left|T_{2}\right|+\left|T_{4}\right|\right) n \\
& \quad-\left|T_{3}\right|-\left|T_{5}\right|-\left|T_{6}\right|
\end{aligned}
$$

i.e. we obtain the same formula as (32) (and (33) respectively). Rearranging terms, we write (41) as

$$
\begin{aligned}
& \left(\left|-T_{1} \lambda^{2}+T_{2} \lambda+T_{3}\right|-\lambda^{3}-K_{1} \lambda^{2}+K_{2} \lambda+K_{3}\right)^{2} \\
& \quad+\left(\left|T_{2} \lambda+T_{3}\right|+T_{1} \lambda^{2}\right)^{2}+\left(\lambda^{2}+K_{1} \lambda\right)^{2} \\
& \quad+\left(T_{2}-T_{3} \lambda\right)^{2}+\left(T_{5}^{2}+2 K_{2}^{2}\right) \lambda^{2}+K_{3}^{2}> \\
& \left(\left|-T_{4} \lambda+T_{5}\right|+T_{6}\right)^{2}+\left(T_{5} \lambda+T_{4}\right)^{2} \\
& +\left(K_{2} \lambda+K_{3}\right)^{2}+\left(T_{2} \lambda+T_{3}\right)^{2}+\left(\lambda^{3}-K_{3}\right)^{2} \\
& +\left(K_{1} \lambda^{2}-K_{2} \lambda\right)^{2}+T_{1}^{2} \lambda^{4}+T_{3}^{2} \lambda^{2}
\end{aligned}
$$

Therefore, the following theorem can be formulated 
Theorem 4. Let $K_{3}-\left|T_{3}\right|-\left|T_{5}\right|-\left|T_{6}\right| \neq 0$ and (43) hold. Then there is no change in stability of $\bar{E}$.

Remark 3. In the special case that $\tau_{1}=\tau_{2}=$ $\tau_{3}$, the characteristic equation (9) becomes

$$
\begin{aligned}
& \chi^{3}+K_{1} \chi^{2}+K_{2} \chi+K_{3}=\ell^{-\chi \tau_{3}}\left(T_{1} \chi^{2}+T_{2} \chi\right. \\
& \left.+T_{3}\right)+\ell^{-2 \chi \tau_{3}}\left(T_{4} \chi+T_{5}\right)+T_{6} \ell^{-3 \chi \tau_{3}} .
\end{aligned}
$$

Hence, this case is a private one of Theorem 4.

Corollary 4.1. If conditions of Theorem 4 are not valid and $\tau_{3}^{\text {bif }}$ is defined as in Theorem 2, then according to Lemma 1 for any $\tau_{3} \in\left[0, \tau_{b}\right)$, there exists a $\tau_{1}^{\text {bif }}\left(\left(\tau_{3}\right)\right)>0\left(\tau_{2}^{\text {bif }}\left(\tau_{3}\right)>\right.$ $0 ; \tau_{4}^{\text {bif }}\left(\tau_{3}\right)>0 ; \tau_{5}^{\text {bif }}\left(\tau_{3}\right)>0$ respectively) such that the steady state $\bar{E}$ of system (2) is unstable when $\tau_{1} \in\left[0, \tau_{1}^{\text {bif }}\left(\tau_{3}\right)\right)\left(\tau_{2} \in\left[0, \tau_{2}^{\text {bif }}\left(\tau_{3}\right)\right) ; \tau_{4} \in\right.$ $\left[0, \tau_{4}^{\text {bif }}\left(\tau_{3}\right)\right) ; \tau_{5} \in\left[0, \tau_{5}^{\text {bif }}\left(\tau_{3}\right)\right)$ respectively), and an Andronov-Hopf bifurcation takes place.

In next Section, we illustrate numerically the existence of the behavior predicted for some values of the rate constants $k_{i}(i=1, \ldots, 6), \gamma_{1}, \gamma_{2}, l$ and the time delays $\tau_{1}, \tau_{2}$ and $\tau_{3}$.

\section{NUMERICAL ANALYSIS}

In the previous section, we proposed the analytical tools and used them for a qualitative analysis of the system, obtaining predictions about dynamics of the system, i.e. the stability and existence of periodic solutions via Andronov-Hopf bifurcation in time delay model (2). In this section, we perform a numerical analysis of model (2), based on the results previously obtained.

Some of the parameter values used in the numerical analysis were selected according to $[4,20$,
$24,29,33]$ in the form

$$
\begin{aligned}
& k_{1}=0.3\left[\min ^{-1}\right], k_{2}=k_{3}=1\left[\min ^{-1}\right], \\
& k_{4}=0.3\left[\min ^{-1}\right], k_{5}=0.5\left[\min ^{-1}\right], \\
& \gamma_{1}=0.1\left[\min ^{-1}\right], \gamma_{2}=0.2\left[\min ^{-1}\right], \\
& \tau_{1} \in[1,8], \tau_{2} \in[12,35] .
\end{aligned}
$$

According to $[6,32]$ proteins (or RNAs) degraded with a probability that depends on their structure because some of the degradation mechanisms involve multiple steps. Therefore, individual protein (or RNA) senesces through time. MiRNAs which are incorporated into the RISC complex, do not degrade with their targets but return to the cytosol a new round of target mRNA repression. It is plausible, however, that miRNA may be degraded after a few cycles of target mRNA binging [13]. Since we do not know the exact values of the average time delay for degradation of miRNA, we set $\tau_{3}$ in large boundaries- from few minutes to few hours and its degradation rate $k_{6} \in[0.05,0.3]$ $\left(\min ^{-1}\right)$. Our model include also one additional parameter for which no values are available and his estimations require further experimental studies. Thus, we assume here that $l=0.1$ in minutes.

In order to compare the predictions with numerical results, the governing equations of the model (2), were solved numerically using MATLAB [18]. In Figure 3, the stable solutions for the concentration of mRNA ( $\left.y_{1}\right)$, the concentration of protein $\left(y_{2}\right)$ and the concentration of miRNA $\left(y_{3}\right)$ are shown for absence of time delay (i.e. $\tau_{1}=\tau_{2}=\tau_{3}=0$ ) - see Fig. 3a, and for $\tau_{1}=$ $\tau_{3}=0, \tau_{2}=12$ - see Fig. $3 \mathrm{~b}$. It is evident that after several physiological acceptance fluctuations, the solution of system (2) approaches a constant value (stable equilibrium state). In other words the system possesses a stable equilibrium state which corresponds to a normal miRNA regulation process. This conclusion is in accordance with the Theorem 2 (Corollary 2.1) proofed in previous Section 3. 


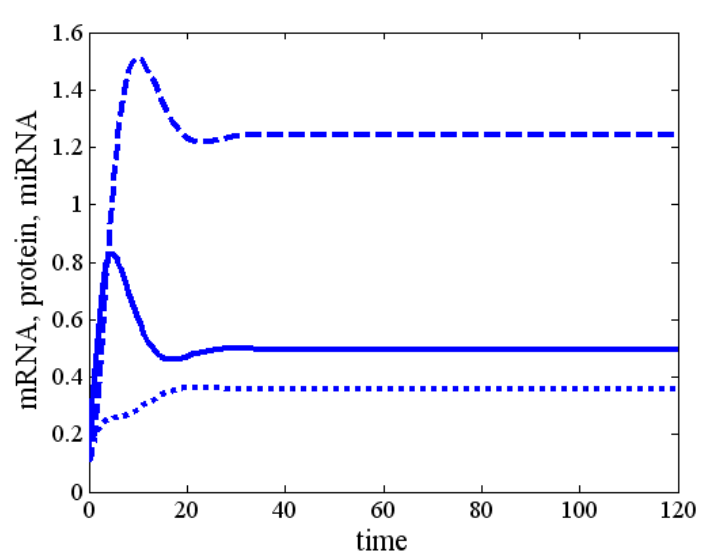

(a)

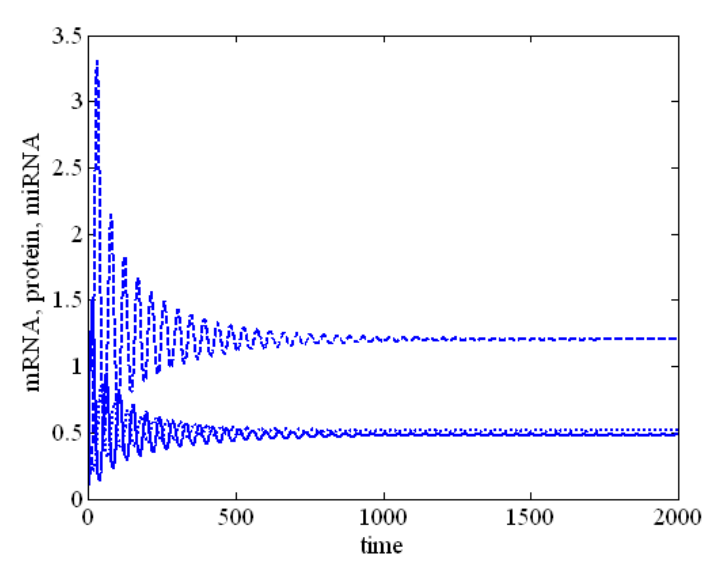

(b)

Fig. 3. Stable regime of system (2) for a) $\tau_{1}=$ $\tau_{2}=\tau_{3}=0$ and $\mathrm{b}$ ) for $\tau_{1}=\tau_{3}=0, \tau_{2}=12$. Model parameters:

$k_{1}=k_{4}=0.3 ; k_{2}=k_{3}=1 ; \gamma_{1}=0.1 ; \gamma_{2}=0.2$; $k_{5}=0.5 ; k_{6}=0.05 ; n_{1}=2 ; l=0.1$.

Figure 4 depicts the cases when $\tau_{2}$ and $\tau_{3}$ are varied. It is seen that for larger values of $\tau_{2}$ (see Fig. 4a) and $\tau_{3}$ (see Fig.4b) than bifurcation one the stable limit cycle (self oscillations) occur and sustained oscillations take place. In other words, in these cases the conditions of Theorem 3 and Theorem 4 are not satisfied and the steady state of system (2) is unstable. From biological point of view, the occurrence of oscillation implies that if the average time delay for degradation of miRNA, $\tau_{3}$, can increase, then the effect of miRNA on gene expression is initially destabilizing. If the average time delay for degradation of miRNA increases further, then the effect of miRNA on gene expression can promote stability- see Fig. $4 c$, and again instability- see Fig. 4d. Thus gene expression follows a cyclic pattern (from stable to unstable behaviour and vice versa) as function of average time delay for degradation of miRNA. This cyclic regime is shown in Figure 5.

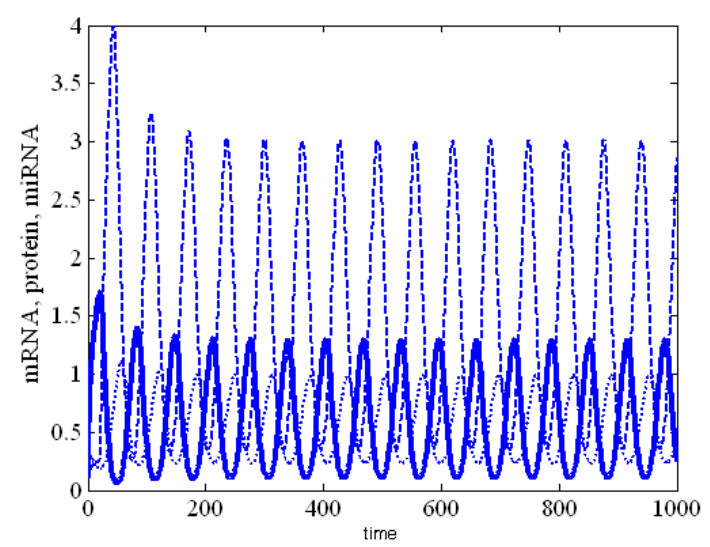

(a)

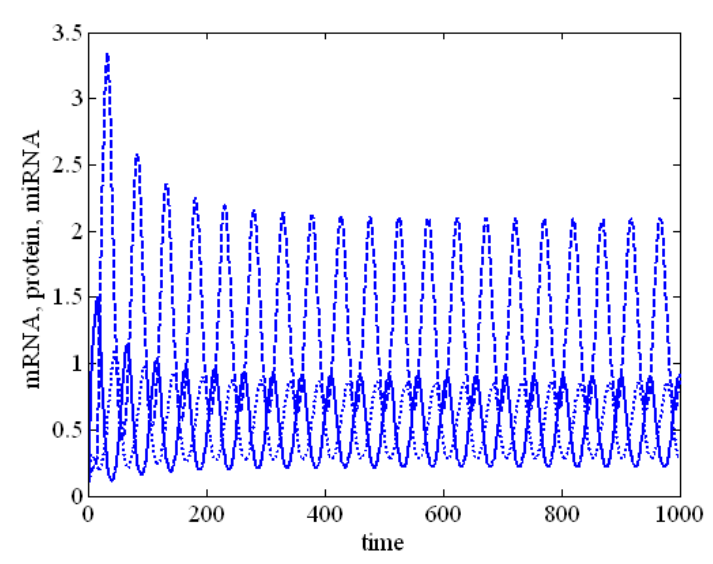

(b) 


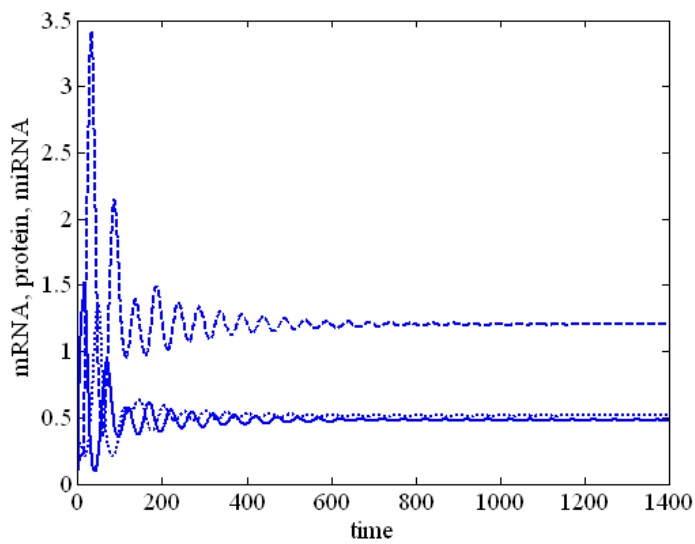

(c)

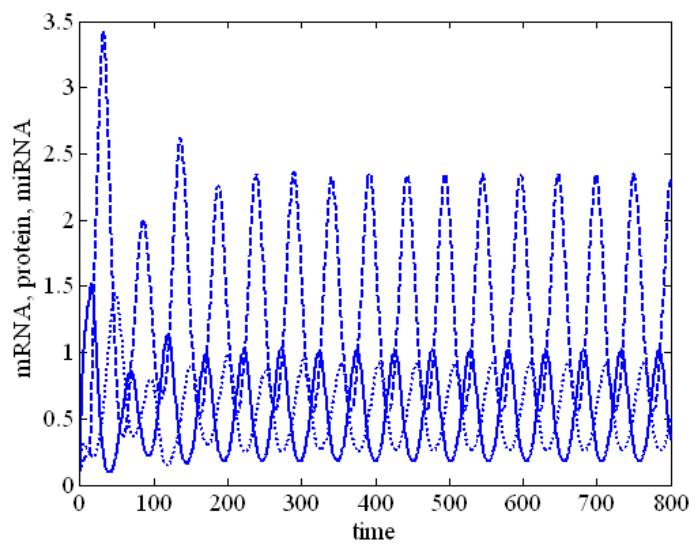

(d)

Fig. 4. Dynamic behaviour of system (2). Unstable regime (periodic oscillations) for $\tau_{1}=\tau_{3}=0$, $\tau_{2}=20(\mathrm{a})$; and $\tau_{1}=0, \tau_{2}=13, \tau_{3}=10(\mathrm{~b})$. Stable regime for $\tau_{1}=1, \tau_{2}=13, \tau_{3}=37$ (c). Unstable regime (sustained oscillations) for $\tau_{1}=1, \tau_{2}=13, \tau_{3}=60(\mathrm{~d})$.

In Figure 5, the stable and unstable zones in the $\left(\tau_{3}, \tau_{1}+\tau_{2}\right)$ parameter space are shown. It is seen that these zones are with different size. It is also interesting to note that in unstable zones sustained oscillations with period one and quasiperiodic motion take place.

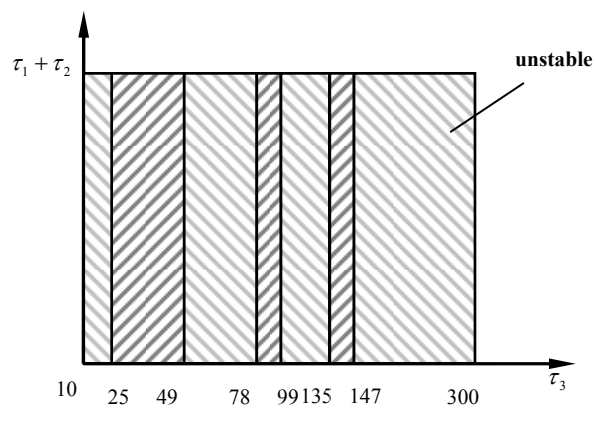

Fig. 5. Stable and unstable zones of system (2) at $\tau_{1}=1, \tau_{2}=13$ and $\tau_{3} \in[10,300]$.

\section{Conclusions}

Gene expression in the human organism is posttranscriptionally regulated by miRNAs. MiRNAs are embedded in complex regulatory networks that involved gene activation, post-translational regulation and protein-protein interactions. Therefore, this makes miRNAs as one of the most abundant classes of regulatory genes in animals. In the present paper we develop a time delay model of a feedback system regulated via miRNA. Our hypothesis (according [33] is that miRNA can participate in the regulation of gene expression by accelerating the degradation of mRNA or by repressing the translation process. The model resulted in three DDEs with three discrete time delays. Since this system is a classical case study, covering several essential features of miRNA and genetic regulatory mechanisms, general conclusions about design principles and role of time delays in the stability of gene circuits can be suggested.

The basic view that time delays $\tau_{1}, \tau_{2}$ and $\tau_{3}$ are a key factor in the dynamic behaviour of the system was confirmed by the analytical calculations and numerical simulations. In more details, under the assumption that an equilibrium exists, we have estimated the length of delays for which local asymptotic stability will be preserved. We have also derived criteria for which no change 
in stability will occur. If system (2) starts with a stable equilibrium, which for some delay(s) becomes unstable, it will likely destabilize by means of an Andronov-Hopf bifurcation leading to small amplitude periodic solutions. Our investigation of such a behaviour is devoted to the use of bifurcation analysis. Particularly, a Hopf bifurcation theorem was employed. From the viewpoint of the qualitative theory of DDEs, time delay(s) appears as a bifurcation parameter on whose values the altered (stable or unstable) behaviour of the model depends. For time delays longer than $\tau_{b}$, the gene expression system regulated by means of miRNA would present sustained oscillations with coupled periodic variations on the concentration of the mRNA, protein and the miRNA. In contrast, a time less than $\tau_{b}$ would provoke damped oscillations around a stable steady state. We can say that in this case time delays have a destabilizing role. From a physiological point of view, the loss of stability might be related to emergence of new configurations in the regulatory gene circuit that could lead the system to a pathological state.

To conclude, mathematical modelling and analysis can enable to understand the mechanism underlying an observed biological process, and at the same time, provide a testable hypothesis for future studies. In this paper we investigate the main processes of the formation of a protein- transcription time (starts with splicing and polyadenylation of the initial transcript), translation time (the timespan from the emergence of mRNA) and average time delay for degradation of miRNA. Thus, our dynamical predictions can be tested in future experiments.

\section{REFERENCES}

[1] A. Andronov, A. Witt and S. Chaikin Theory of Oscillations, Addison-Wesley, 1966.

[2] O. Arino, M. Hbib and E. AitDads, Delay Differential Equations and Applications, Springer, 2006.

[3] M. Bueno, I. Peres de Castro, M. Malumbres, Control of cell proliferation pathways by microRNAs, Cell Cycle 7(20) (2008) 3143-3148.

[4] H. de Jong, Modeling and simulation of gene regulatory systems: a literature review, J. of Comp. Biol. 9(1) (2002) 67-103.
[5] H. Freedman, and V. Rao, The trade-off between mutual interference and time lags in predator-prey systems. Bull. Math. Biol. 45(6) (1983) 991-1004.

[6] A. Hershko and A. Ciechanover, The ubiquitin system. Annu. Rev. Biochem. 67 (1998) 425-479.

[7] R. Hilborn, Chaos and Nonlinear Dynamics: an Introduction for Sciencists and Engineers, Second edtion, Oxford University Press, 2011.

[8] C. Kanellopoulou and S. Monticelli, A role for microRNAs in the development of the immune system and in the pathogenesis of cancer, Seminars in Cancer Biol., 18 (2008) 79-88.

[9] Q. Khan and D. Greenhalgh, Hopf bifurcation in epidemic models with a time delay in vaccination. IMA J. of Math. Applied in Medicine and Biology 16 (1999) 113-142.

[10] Q. Khan, Hopf bifurcation in multiparty political systems with time delay in switching. Appl. Math. Lett. 13 (2000) 45-52.

[11] F.M. Khan, U. Schmitz, S. Nikolov and et al., Hybrid modeling of the crosstalk between signaling and transcriptional networks using ordinary differential equations and multi-valued logic, BBA (Biochimica et Biophysica Acta)- Proteins and Proteomics, 1844(1) (2014) 289-298.

[12] R. Khanin and V. Vinciotti, Computational modeling of post-transcriptional gene regulation by microRNAs, J. Comput Biol. 15(3) (2008) 305-316.

[13] R. Khanin and D. Higham, 2010. Mathematical and computational modelling of post-transcriptional gene regulation by miRNAs. In: MiRNA profiling in Cancer. A Bioinformatics Perspective, Pan Stanford Publishing Pte. Ltd. Chapter 10 (2010) 197-216.

[14] G. Korn and T. Korn, Mathematical Handbook for Scientists and Engineers. McGraw-Hill, 1968.

[15] H. Lodish, A. Berk, P. Matsudaira and et al., Molecular Cell Biology, Freeman and Company, 2004.

[16] J. Lytle, Th. Yario and J. Steitz, Target mRNAs are repressed as efficiently by microRNA-binding sites in the 5'UTR as in the 3'UTR, PNAS 104(23) (2007) 96679672 .

[17] J. Marsden and M. McCracen, The Hopf Bifurcation and its Applications, Springer-Verlag, 1976

[18] Matlab, The MathWorks, Inc. 2010. http://www.mathworks.com/

[19] R. May, Stability and Complexity in Model Ecosystems. Princeton University Press, 1973.

[20] N. Monk, Oscillatory expression of Hes1, p53, and NFkappaB driven by transcriptional delay, Curr. Biol. 13 (2003) 1409-1413.

[21] D. Nicholl, An Introduction to Genetic Engineering. Cambridge Univ. Press, 2008. http://dx.doi.org/10.1017/CBO9780511800986

[22] S. Nikolov, J. Vera, U. Schmitz and O. Wolkenhauer, A model-based strategy to investigate the role of microRNA regulation in cancer signalling networks. Theory in Biosciences 130(1) (2011) 55-69. 


\section{S. Nikolov, Modelling and Analysis of miRNA Regulation}

[23] S. Nikolov, Stability and Andronov-Hopf bifurcation of a system with three time delays, Journal of Mathematics, 2013 (2013) art. ID 347071(11 pages).

[24] K. Rateitschak and O. Wolkenhauer, Intracellular delay limits cyclic changes in gene expression, Math. Biosci. 205 (2007) 163-179.

[25] Sh. Ruan and J. Wei, On the zeros of transcendental functions with applications to stability of delay differential equations with two delays, Dynamics of Continuous, Discrete and Impulsive Systems, Series A: Mathematical Analysis 10 (2003) 863-874.

[26] A. Sensse and M. Eiswirth, Feedback loops for chaos in activator-inhibitor systems, The Journal of Chemical Physics 122 (2005) 044516.

[27] L. Shilnikov, A. Shilnikov, D. Turaev and L. Chua, Methods of Qualitative Theory in Nonlinear Dynamics. Part II, World Scientific, 2001.

[28] P. Smolen, D. Baxter and J. Byrne, Effects of macromolecular transport and stochastic fluctuations on dynamics of genetic regulatory systems, Am. J. Physiol. 277(4 Pt 1) (1999) C777-C790.

[29] I. Swameye, T. Mueller, J. Timmer, and et al., Identification of nucleocytoplasmic cycling as a remote sensor in cellular signaling by data-based modelling, PNAS 100 (2003) 1028-1033.

[30] I.A. Swinburne, P.A. Silver, Intron delays and transcriptional timing during development. Dev Cell. 14(3) (2008) 324-330.

[31] J. Tsang, J. Zhu and A. van Ondenaarden, MicroRNAmediated feedback and feedforward loops are recurrent networks motifs in mammals, Mol. Cell., 26(5) (2007) 753-767. http://dx.doi.org/10.1016/j.molcel.2007.05.018

[32] X. Wang, Y. Li, X. Xu and Y. Wang, Toward a system level understanding of microRNA pathway via mathematical modelling, BioSystems 100 (2010) 31-38.

[33] Zh. Xie, H. Yang, W. Liu, and M. Hwang, The role of microRNA in the delayed negative feedback regulation of gene expression, Bioch. and Bioph. Research Comm. 358 (2007) 722-726.

[34] S. Zeiser, H. Liebscher, H. Tiedemann, and et al., Number of active transcription factors binding sites is essential for the Hes7 oscillator. Theor. Biol. Med. Model 23 (2006) 11.

[35] S. Zeiser, O. Rivera, C. Kuttler and et al., Oscillations of Hes7 caused by negative autoregulation and ubiquiti nation, Comput. Biol. Chem. 32 (2008) 48-52. 